\title{
ON THE DYNAMIC SCALING BEHAVIOUR OF SOLUTIONS TO THE DISCRETE SMOLUCHOWSKI EQUATIONS
}

\author{
by F. P. DA COSTA
}

(Received 6th December 1994)

In this paper we generalize recent results of Kreer and Penrose by showing that solutions to the discrete Smoluchowski equations

$$
\dot{c}_{j}=\sum_{k=1}^{j-1} c_{j-k} c_{k}-2 c_{j} \sum_{k=1}^{\infty} c_{k}, j=1,2, \ldots
$$

with general exponentially decreasing initial data, with density $\rho$, have the following asymptotic behaviour

$$
\lim _{\substack{j, t \rightarrow \infty \\ \xi j / f \text { fixed } \\ j \in g}} t^{2} c_{j}(t)=\frac{q}{\rho} e^{-\xi / \rho}
$$

where $\mathscr{J}=\left\{j: c_{j}(t)>0, t>0\right\}$ and $q=\operatorname{gcd}\left\{j: c_{j}(0)>0\right\}$.

1991 AMS Subject Classification: 82C22, 34D05, 12D10

\section{Introduction}

The discrete Smoluchowski equations are a model for the kinetics of irreversible cluster growth, in which $j$-clusters (clusters with $j$ particles) can coagulate with $k$-clusters to give $(j+k)$-clusters, but the reverse process of cluster fragmentation does not occur.

Denoting by $c_{j}=c_{j}(t) \geqq 0$ the concentration of a $j$-cluster at time $t \geqq 0$, the equations are

$$
\dot{c}_{j}=\sum_{k=1}^{j-1} a_{j-k, k} c_{j-k} c_{k}-2 c_{j} \sum_{k=1}^{\infty} a_{j, k} c_{k}, j=1,2, \ldots
$$

where $a_{j, k}=a_{k, j} \geqq 0$ are the rate coefficients for the coagulation reactions, and the first sum is defined to be zero if $j=1$. Physically, the quantity $\rho(t):=\sum_{j=1}^{\infty} j c_{j}(t)$ is the total density of the system at time $t$, and should be finite. This is reflected, mathematically, in the choice of the Banach space of finite density sequences, $X=\left\{c=\left(c_{j}\right):\|c\|:=\right.$ $\left.\sum_{j=1}^{\infty} j\left|c_{j}\right|<\infty\right\}$, as the natural space for the study of (1). The mathematical theory of 
these equations, as well as that of more general equations describing the kinetics of reversible cluster growth, has been the subject of several papers in recent years, and questions about existence and uniqueness of solutions $[1,2,15]$, and their asymptotic behaviour $[2,4,5,13,14]$ have been studied.

There are still a number of important open problems concerning the asymptotic behaviour of solutions to (1). It is easy to prove that, under rather general conditions on the coefficients $a_{j, k}$ all global solutions to (1) converge, in the weak* topology of $X$, to the zero solution as $t \rightarrow \infty$, [5, Theorem 4.3]. On the other hand, the asymptotic behaviour of solutions in the strong (norm) topology of $X$ is not yet completely understood, particularly when the coagulation coefficients $a_{j, k}$ grow rapidly with $j$ and $k$, (see $[4,13]$ and references therein). For slowly growing coefficients, namely for $a_{j, k} \sim(j k)^{\alpha}, \alpha \leqq 1 / 2$, it has been proved, [1], that the solution of (1) with initial condition $c(0)$ satisfies $\|c(t)\|=\rho:=\|c(0)\|$ for all $t \geqq 0$.

This different asymptotic behaviour of solutions in the strong and in the weak* topologies of $X$ is analogous to what happens in the Becker-Döring and Generalized Becker-Döring equations for supercritical initial data, i.e., initial data with density $\rho>\rho_{s}$ where $\rho_{s}$ is a constant depending only on the coefficients of the equation $[2,5,14]$. A problem that naturally arises in this context is to elucidate how is the total density $\rho$ distributed among the various clusters and how does this distribution evolve with time. As pointed out by Ball et al. [3] this problem is, in a certain sense, related to the question of how the energy of a dissipative infinite dimensional dynamical system is distributed among the various modes as $t \rightarrow \infty$.

A particular case of this type of problems occurs in the physics literature under the names of similarity solutions, [7, Section 6.4], self-preserving size distribution, [10], or dynamic scaling behaviour, $[8,9]$. The conjecture is that for sufficiently large times and cluster sizes the shape of the distribution of the total density among the $j$-clusters approaches a self-similar profile independent of that of the initial data; more precisely, for sufficiently large $t$ and $j$ the solution $c_{j}(t)$ approaches $t^{-\alpha} \tilde{c}\left(j t^{-\beta}\right)$ for some positive constants $\alpha$ and $\beta$, and for some function $\tilde{c}: \mathbb{R}^{+} \rightarrow \mathbb{R}^{+}$.

Recently Kreer and Penrose obtained a mathematically rigorous proof of this conjecture, in the case where $a_{j, k}=1$ for all $j$ and $k$, and the initial data are exponentially decreasing and satisfy $c_{1}(0)>0,[12]$.

They use the generating function

$$
\Phi(z, t):=\sum_{j=1}^{\infty} c_{j}(t) z^{j}
$$

defined for all $t \geqq 0$ and $z \in \overline{B_{1}}:=\{z \in \mathbb{C}:|z| \leqq 1\}$, in order to obtain an integral expression for $c_{j}(t)$ : one can easily prove that $\Phi(z, t)$ satisfies a differential equation (see Proposition 4 below) that can be integrated to give

$$
\Phi(z, t)=t^{-2} \frac{1}{N_{0}+t^{-1}} \frac{\phi(z)}{N_{0}+t^{-1}-\phi(z)}
$$


where $N_{0}:=\sum_{j=1}^{\infty} c_{j}(0)$ and $\phi(z):=\Phi(z, 0)=\sum_{j=1}^{\infty} c_{j}(0) z^{j}$. Since, for each $t \geqq 0, \Phi(z, t)$ is analytic in $B_{1}$ one can use the Cauchy integral formula in (3) to obtain

$$
t^{2} c_{j}(t)=\frac{1}{2 \pi i} \frac{1}{N_{0}+t^{-1}} \oint_{\gamma_{0}} \frac{1}{z^{j+1}} \frac{\phi(z)}{N_{0}+t^{-1}-\phi(z)} d z
$$

where $\gamma_{0}=\left\{z \in \mathbb{C}:|z|=r_{0}\right\}$ for some $r_{0} \in(0,1)$.

The fundamental observation of [12], that makes possible the asymptotic evaluation of (4), is the following: if the initial data $\left(c_{j}(0)\right)$ satisfies

$$
0 \leqq c_{j}(0) \leqq A(1+\Delta)^{-j}
$$

for some positive constants $A$ and $\Delta$, independent of $j$, then $\phi(z)$ is analytic in the ball $|z|<1+\Delta$ and, for each $t \geqq 0$, so is $\Phi(z, t)$, except for poles at the zeros of the denominator of the expression in the right-hand side of (3). Assuming $c_{1}(0)>0$ and denoting $t^{-1}$ by $\tau$, Kreer and Penrose proved that $N_{0}+\tau-\phi(z)$ has only one zero, $z_{1}(\tau)$, that converge to the unit circle $\partial B_{1}$ as $\tau \rightarrow 0$, all the other zeros being bounded away form $\overline{B_{1}}$ as $\tau \rightarrow 0$. This allows (4) to be asymptotically evaluated by considering the integral on a larger circle $\gamma_{2}$ such that $z_{1}(\tau)$ is the only pole in its interior, minus the integral on a small circle $\gamma_{1}$ that contains $z_{1}(\tau)$ in its interior for all sufficiently small values of $\tau$. The integral over $\gamma_{2}$ can be shown to converge to zero uniformly as $j, t \rightarrow \infty$ with $j / t$ bounded, and the integral over $\gamma_{1}$ can be evaluated using the residue theorem, and the limit as $t \rightarrow \infty$ with $\xi=j / t$ fixed can be computed from the knowledge of the behaviour of $z_{1}(\tau)$ as $\tau \rightarrow 0$.

Using the above method, Kreer and Penrose concluded that

$$
\lim _{\substack{j, t \rightarrow \infty \\ \xi=j / t \text { fixed }}} t^{2} c_{j}(t)=\frac{1}{\rho} e^{-\xi / \rho}
$$

uniformly for $\xi$ in compact subsets of $\mathbb{R}^{+},[12$, Theorem III].

It is argued in [12] that one should not expect this type of behaviour if the condition $c_{1}(0)>0$ is not fulfilled. In support of this claim the problem with initial data $c_{j}(0)=\delta_{j, m}$, for some integer $m>1$, is considered. For this initial condition the solution can be explicitly computed and is

$$
t^{2} c_{j}(t)= \begin{cases}\left(1+t^{-1}\right)^{-1-j / m} & \text { if } j / m \in \mathbb{N} \\ 0 & \text { otherwise }\end{cases}
$$

and from this one concludes that 
$\lim _{\substack{j, t \rightarrow \infty \\ \xi=j / t \text { fixed }}} t^{2} c_{j}(t)= \begin{cases}e^{-\xi / m} & \text { if } j \rightarrow \infty \text { through a sequence of multiples of } m \\ 0 & \text { if } j \rightarrow \infty \text { through a sequence of integers not multiples of } m .\end{cases}$

Now, what this example actually shows is that the non-zero components of the solution do, in fact, exhibit a dynamic scaling behaviour in the sense that if the limit is computed using only those $j$ s for which $c_{f}(t)>0$ then a limit function analogous to the cases with $c_{1}(0)>0$ is obtained. Another objection to the exclusion of initial data with $c_{1}(0)=0$ is the following: suppose the initial condition $\left(c_{f}(0)\right)$ is such that $c_{1}(0)=0$ but there exists two coprime numbers $p_{1}$ and $p_{2}$ such that $c_{p 1}(0), c_{p 2}(0)>0$. Then, the positivity result in [6] (see Proposition 3 below) and results in elementary number theory (see, e.g., [11, page 277]) imply that there exists a positive integer $m_{0}$ such that $c_{j}(t)>0$ for all $j>m_{0}$ and all $t>0$. Thus, in this case, it is natural to ask if the limit

$$
\lim _{\substack{j, t \rightarrow \infty \\ \xi=j / t \text { fixed }}} t^{2} c_{j}(t)
$$

exists, without having to impose any restrictions on the set of $j$ s to be used in its computation.

In this paper we extend the results of [12] to general exponentially decreasing initial data, proving that an expression similar to (6) holds even for initial data with $c_{1}(0)=0$. We use the Kreer-Penrose method and, as is possibly clear from the discussion above, the key to get the result without assuming $c_{1}(0)>0$ is a more detailed analysis of the zeros of

$$
F(z, \tau):=N_{0}+\tau-\phi(z)
$$

The final result (Theorem 1) is the following:

If the initial data $\left(c_{f}(0)\right)$ satisfies $(5)$ then

$$
\lim _{\substack{j, t \rightarrow \infty \\ \xi j / \text { fixed } \\ j \in g}} t^{2} c_{j}(t)=\frac{q}{\rho} e^{-\xi / \rho},
$$

where $\mathscr{J}$ is the set of subscripts $j$ for which $c_{f}(t)>0$ (which is an infinite set independent 
of $t$ for $t>0$, see below), and $q=\operatorname{gcd}\left\{j: c_{j}(0)>0\right\}$. It is clear that this expression reduces to (6) when $c_{1}(0)>0$ (for which $q=1$ ), and to (7) if $c_{j}(0)=\delta_{j, m}$ (for which $q=\rho=m$ ).

The paper is organized as follows:

In Section 2 we present some preliminaries: basic results on existence, uniqueness, regularity, asymptotic behaviour, and positivity properties of solutions are quoted from previous work on these equations. Moreover, we prove an auxiliary result on the greatest common divisor of a countable set of positive integers that will be needed afterwards.

In Section 3 we study the zeros of $F(z, \tau)=N_{0}+\tau-\phi(z)$ for $\tau$ in a neighbourhood of the origin.

Finally, in Section 4 we prove that the dynamic scaling behaviour (9) holds.

\section{Preliminaries}

Throughout the paper we assume $a_{j, k}=1$ for all $j$ and $k$. System (1) becomes

$$
\dot{c}_{j}=\sum_{k=1}^{j-1} c_{j-k} c_{k}-2 c_{j} \sum_{k=1}^{\infty} c_{k}, j=1,2, \ldots
$$

The following two Propositions are immediate consequences of results in $[1,5]$ :

Proposition 1. $[1,5]$ For every $c_{0}=\left(c_{0 j}\right) \in X^{+}:=X \cap\left\{\left(c_{j}\right): c_{j} \geqq 0\right\}$, system (10) has a unique solution $c \in \mathscr{C}^{0}\left(0, \infty ; X^{+}\right)$such that $c(0)=c_{0}$. The solution $c$ satisfies: $\|c(t)\|=\rho:=$ $\left\|c_{0}\right\|$ for every $t \geqq 0$; the series $\sum_{j} j c_{j}(t)$ is uniformly convergent on compact sets of $[0, \infty)$; each component $c_{j}$ is continuously differentiable on $\mathbb{R}^{+}$; and for all $t \geqq 0$ and all $p \geqq 1$, the series $\sum_{j} j^{p} c_{j}(t)$ is convergent iff $\sum_{j} j^{p} c_{0 j}$ converges.

Proposition 2. [5] The zero solution of (10) is globally asymptotically weak* stable in $X^{+}$, i.e., for all initial data $c_{0} \in X^{+}$the unique solution of $(10)$ with $c(0)=c_{0}$ satisfies

$$
\sup _{t \in[0, \infty)}\|c(t)\|<\infty \text { and } c_{j}(t) \rightarrow 0 \text { as } t \rightarrow \infty \text {, for every } j \text {. }
$$

For the precise characterization of the positivity properties of solutions we need to introduce some notation: being $c(t)=\left(c_{,}(t)\right)$ any solution of $(10)$, let

$$
\begin{gathered}
\mathscr{J}(t)=\left\{j \in \mathbb{N}: c_{j}(t)>0\right\} \\
P=\mathscr{J}(0) \\
\operatorname{span}_{\mathbb{N}_{0}}(P)=\left\{j=\sum_{i} n_{i, j} p_{i}: p_{i} \in P, n_{i, j} \in \mathbb{N}_{0}, \text { and } \max _{i} n_{i, j}>0\right\} .
\end{gathered}
$$


Then we have the following

Proposition 3. [6] Let $c_{0} \in X^{+}$and let $c$ be the solution of (10) with initial condition $c(0)=c_{0}$. Then, for all $t>0, \mathscr{J}(t)=\mathscr{J}$ is independent of $t$ and is given by $\mathscr{J}=\operatorname{span}_{N_{0}}(P)$.

Define the 0th moment of a solution as $N(t)=\sum_{j=1}^{\infty} c_{j}(t)$ and the generating function $\Phi(z, t)$ by $(2)$, then

Proposition 4. [12, Proposition I] Let $c$ be the solution of (10) with initial condition $c_{0} \in X^{+}$. Then $N(t)$ and $\Phi(z, t)$ satisfy the differential equations

$$
\begin{gathered}
\frac{d N}{d t}=-N^{2} \\
\frac{\partial \Phi}{\partial t}=\Phi^{2}-2 N \Phi
\end{gathered}
$$

with initial data $N(0)=N_{0}:=\sum_{j=1}^{\infty} c_{0 j}$ and $\Phi(z, 0)=\phi(z):=\sum_{j=1}^{\infty} c_{0 j} z^{j}$, respectively.

For the study of the zeros of $F(z, \tau)$ we need the following result:

Proposition 5. Let $M=\left\{m_{1}, m_{2}, \ldots\right\}$ be a countable subset of $\mathbb{N}$ and let $q=$ $\operatorname{gcd}\left(m_{1}, m_{2}, \ldots\right)$. Then, there exists a positive integer $n_{0}$ such that $\operatorname{gcd}\left(m_{1}, m_{2}, \ldots, m_{n 0}\right)=q$.

Proof. Let $\mu_{j}$ be the number of divisors of $m_{j}$ and let $d_{k}^{(j)}, k=1, \ldots, \mu_{j}$, denote the divisors of $m_{j}$. Then

Since

$$
q=\max \bigcap_{j=1}^{\infty} \bigcup_{k=1}^{\mu_{j}}\left\{d_{k}^{(j)}\right\}
$$

we have

$$
\bigcap_{j=1}^{n} \bigcup_{k=1}^{\mu_{j}}\left\{d_{k}^{(j)}\right\} \supseteq \bigcap_{j=1}^{n+1} \bigcup_{k=1}^{\mu_{j}}\left\{d_{k}^{(j)}\right\}
$$

$$
q_{n}:=\max \bigcap_{j=1}^{n} \bigcup_{k=1}^{\mu_{j}}\left\{d_{k}^{(j)}\right\} \geqq \max \bigcap_{j=1}^{n+1} \bigcup_{k=1}^{\mu_{j}}\left\{d_{k}^{(j)}\right\}=: q_{n+1} \geqq q .
$$

Thus, the sequence $\left(q_{n}\right)$ is monotonic nonincreasing and bounded below. Hence, it converges and so it must be constant and equal to $q$ for sufficiently large $n$.

\section{The zeros of $F(z, \tau)$}

With $P$ defined by (12) denote the elements of $P$ by $p_{1}, p_{2}, \ldots$ Assume $c_{0}=\left(c_{0 j}\right)$ 
satisfies (5). Then $\phi(z):=\sum_{j=1}^{\infty} c_{0 j} z^{j}=\sum_{p_{j} \in P} c_{0 p_{j}} z^{p^{j}}$ is defined and analytic in the ball $B_{1+\Delta}=\{z \in \mathbb{C}:|z|<1+\Delta\}$. Consider the function $F: B_{1+\Delta} \times[0, \infty) \rightarrow \mathbb{C}$ defined by (8). We start the study of the zeros of $F$ with the case $\tau=0$.

Proposition 6. With the above assumption and definitions we have:

(i) All zeros of $F(z, 0)$ lie in $B_{1+\Delta} \backslash B_{1}$.

(ii) Let $q=\operatorname{gcd}\left(p_{1}, p_{2}, \ldots\right)$. Then $F(z, 0)$ has exactly $q$ zeros on the unit circle $\partial B_{1}$, which are the $q^{\text {th }}$ roots of unity, and all of them are simple.

Proof. (i) Let $f$ be the restriction of $F(\cdot, 0)$ to $[0, \infty)$. Let $\tau_{1}:=-f(1+\Delta) \in \mathbb{R}^{+} \cup\{\infty\}$. Since $f(0)=N_{0}>0, \quad f(x) \rightarrow-\tau_{1}<0 \quad$ as $\quad x \uparrow 1+\Delta, \quad$ and $\quad f^{\prime}(x)=-\phi^{\prime}(x)=$ $-x^{-1} \sum_{p_{j} \in P} p_{j} c_{0_{p j}} x^{p_{j}}<0$ for all $x \in(0,1+\Delta)$, we conclude that $f$ has only one zero in $(0,1+\Delta)$, which, by the definition of $f$, is clearly $x=1$. Now suppose $z \in \mathbb{C}$ is a zero of $F(z, 0)$. Then, since $N_{0}=|\phi(z)| \leqq \phi(|z|)$, we have $f(|z|)=F(|z|, 0) \leqq 0$, and thus $|z| \geqq 1$.

(ii) Let $\zeta:=z^{q}$ and $m_{j}:=p_{j} / q$. Clearly $\operatorname{gcd}\left(m_{1}, m_{2}, \ldots\right)=1$. Define $\psi(\zeta):=N_{0}-$ $\sum_{p_{j} \in P} c_{0_{p_{j}}} \zeta^{m_{j}}$. We have $\psi(\zeta(z))=F(z, 0)$. Being interested in the case $|\zeta|=|z|=1$, let $\zeta=e^{i \theta}$. A complex number $\zeta$ is a zero of $\psi$ iff $N_{0}-\sum_{j} c_{0 p_{j}} \zeta^{m_{j}}=0$, i.e.,

$$
\sum_{j} c_{0 p_{j}}\left(1-\cos \left(m_{j} \theta\right)\right)=0
$$

and, since $c_{0 p_{j}}>0$ for all $j$, we must have

$$
m_{j} \theta=0 \bmod 2 \pi
$$

Clearly $\theta=0 \bmod 2 \pi$ is a solution of (16). We prove it is the only solution. Observe that (16) is an infinite system of equations if $P$ is infinite. We need only to show that for some finite subsystem the only solution is $\theta=0 \bmod 2 \pi$. By Proposition 5 we know that, for some integer $n_{0}, \operatorname{gcd}\left(m_{1}, m_{2}, \ldots, m_{n 0}\right)=1$. Consider the following finite subsystem of (16):

$$
m_{j} \theta=0 \bmod 2 \pi, j \in\left\{1, \ldots, n_{0}\right\}
$$

Suppose there exist $k$ and $l$ such that $m_{k}$ and $m_{l}$ are coprimes, i.e. $\operatorname{gcd}\left(m_{k}, m_{l}\right)=1$. Then

$$
\begin{aligned}
& m_{k} \theta=2 \alpha \pi \\
& m_{l} \theta=2 \beta \pi
\end{aligned}
$$

for some integers $\alpha$ and $\beta$. Thus $\beta=m_{l} \alpha / m_{k}$ and since $m_{k}$ does not divide $m_{l}$ it follows that there exists an integer $r$ such that $\alpha=r m_{k}$, which means that (18) can be written as $\theta=2 r \pi$, proving the result. If there are no pairwise coprimes in $\left\{m_{1}, m_{2}, \ldots, m_{n_{0}}\right\}$ we can argue as follows: take two equations from (17), for instance, $m_{1} \theta=0 \bmod 2 \pi$ and $m_{2} \theta=0 \bmod 2 \pi$ and let $g_{1}=\operatorname{gcd}\left(m_{1}, m_{2}\right)$. Write $m_{1}=g_{1} r_{1}, m_{2}=g_{1} r_{2}$. As $\operatorname{gcd}\left(r_{1}, r_{2}\right)=1$ we 
can repeat the argument above to conclude that $g_{1} \theta=0 \bmod 2 \pi$. Proceeding in the same way for pairs of equations with $j=3$ and $j=4$, etc, we obtain another system of equations for $\theta$. The crucial fact here is that this new system has fewer equations than (17). To be more precise, let $h(x)=[(x+1) / 2]$, where $[y]$ denotes the integer part of $y$, and define

$$
\begin{gathered}
g_{i}^{(1)}=\operatorname{gcd}\left(m_{2 i-1}, m_{2 i}\right) \text { for } i=1,2, \ldots, h\left(n_{0}\right)-1 \\
g_{h\left(n_{0}\right)}^{(1)}= \begin{cases}\operatorname{gcd}\left(m_{n_{0}-1}, m_{n_{0}}\right) & \text { if } n_{0} \text { is even } \\
m_{n_{0}} & \text { if } n_{0} \text { is odd, }\end{cases}
\end{gathered}
$$

We have $\operatorname{gcd}\left(g_{1}^{(1)}, g_{2}^{(1)}, \ldots, g_{h\left(n_{0}\right)}^{(1)}\right)=\operatorname{gcd}\left(m_{1}, m_{2}, \ldots, m_{n_{0}}\right)=1$, and if $\theta=0 \bmod 2 \pi$ is the only solution of the equations

$$
g_{j}^{(1)} \theta=0 \bmod 2 \pi, j \in\left\{1, \ldots, h\left(n_{0}\right)\right\},
$$

then it is also the only solution of (17). If at least two of the $g_{j}^{(1)} s$ are pairwise coprimes we argue as previously. Otherwise, we can recursively define

$$
\begin{aligned}
g_{i}^{(l)} & =\operatorname{gcd}\left(g_{2 i-1}^{(l-1)}, g_{2 i}^{(l-1)}\right) \text { for } i=1,2, \ldots, h^{(l)}\left(n_{0}\right)-1 \\
g_{h}^{(l)}\left(n_{0}\right) & = \begin{cases}\operatorname{gcd}\left(g_{h^{(i-1)}}^{(l-1)}\left(n_{0}\right)-1\right. & , g_{h^{(l-1}}^{(l-1)}\left(n_{0}\right) \\
g_{h^{(l-1)}\left(n_{0}\right)}^{(i-h} & \text { if } h^{(l-1)}\left(n_{0}\right) \text { is even } \\
\end{cases}
\end{aligned}
$$

where $h^{(l)}\left(n_{0}\right)=h\left(h^{(l-1)}\left(n_{0}\right)\right)$ for $l \geqq 2$, and consider the equations

$$
g_{j}^{(l)} \theta=0 \bmod 2 \pi, j \in\left\{1, \ldots, h^{(l)}\left(n_{0}\right)\right\} .
$$

Let $\eta$ be the nonnegative integer such that $n_{0} \in\left(2^{\eta}, 2^{\eta+1}\right]$. We need only to apply this procedure at most $\eta$ times in order to obtain a pair of coprimes. In the worst possible case we end up with the coprimes $g_{1}^{(\eta)}$ and $g_{2}^{(\eta)}$ and we can now solve the corresponding system $(20)$ and obtain $\theta=0 \bmod 2 \pi$ as its unique solution.

Hence, $\zeta=1$ is the only zero of $\psi$ in the unit circle and, consequently, the only zeros of $F(z, 0)$ in the unit circle are the $q^{\text {th }}$ roots of unity, $\omega_{q}^{k}=\exp (2 \pi i k / q), k=1, \ldots, q$.

Furthermore, for $z \neq 0$,

$$
F^{\prime}(z, 0)=-z^{-1} \sum_{p_{j} \in P} p_{j} c_{0 p_{j}} z^{p_{j}}
$$

and thus, for all $k=1, \ldots, q$,

$$
F^{\prime}\left(\omega_{q}^{k}, 0\right)=-\omega_{q}^{-k} \sum_{p_{j} \in P} p_{j} c_{0 p} \omega_{q}^{p_{j} k}=-\omega_{q}^{-k} \sum_{m_{j q \in P}} m_{j} q c_{0 m_{j q}}\left(\omega_{q}^{q}\right)^{m_{j} k}=-\omega_{q}^{-k} \rho \neq 0 .
$$


This concludes the proof.

We now turn to the study of $F(z, \tau)$ with $\tau>0$. The following result generalizes Lemma II of [12]:

Proposition 7. With the assumptions of Proposition 6 the following holds true:

(i) For all sufficiently small $\tau \in\left(0, \tau_{1}\right)$ there exists $q$ simple zeros of $F(z, \tau)$, denoted $z_{k}(\tau)$ with $k=1, \ldots, q$, satisfying $\left|z_{k}(\tau)\right|>1$ and

$$
z_{k}(\tau)=\omega_{q}^{k}\left(1+\frac{1}{\rho} \tau+R^{(2)}(\tau)\right)
$$

where $R^{(2)}(\tau) \sim \mathcal{O}\left(\tau^{2}\right)$ as $\tau \rightarrow 0$.

(ii) All other zeros of $F(z, \tau), z_{k}(\tau), k=q+1, q+2, \ldots$, lie outside $\overline{B_{1}}$ and are uniformly bounded away from it as $\tau \rightarrow 0$.

Proof. We start by studying the general location of the zeros. Let $z \in \mathbb{C}$ be a zero of $F(\cdot, \tau)$. Then $N_{0}+\tau=|\phi(z)|$, and, since $\tau>0$ and $|\phi(z)| \leqq \phi(|z|)$, we conclude that

$$
f(|z|)=F(|z|, 0)=N_{0}-\phi(|z|)<N_{0}+\tau-|\phi(z)|=0,
$$

where $f$ was defined in the proof of Proposition 6. This inequality implies $|z|>1$. We now prove statements (i) and (ii) separately.

(i) From the last part of the proof of Proposition 6 we can appy an implicit function theorem to $F(z, \tau)=0$ at $\left(\omega_{q}^{k}, 0\right)$ to conclude that for all sufficiently small $\tau$ there exist functions $z_{k}(\tau)$ such that $F\left(z_{k}(\tau), \tau\right) \equiv 0$ and $z_{k}(\tau) \rightarrow \omega_{q}^{k} \in \partial B_{1}$ as $\tau \rightarrow 0$. By (21) there exists a $\tau_{2} \in\left(0, \tau_{1}\right)$ such that, for all $\tau \in\left[0, \tau_{2}\right),(\partial F / \partial z)\left(z_{k}(\tau), \tau\right) \neq 0$. Furthermore, we can differentiate $F\left(z_{k}(\tau), \tau\right) \equiv 0$ with respect to $\tau$ at $\tau=0$ obtaining (22).

(ii) For the other zeros of $F(z, \tau)$ we argue as in [12]: Being $F(z, 0)$ analytic in $B_{1+\Delta}$ it has only a finite number of zeros inside every ball $B_{1+\Delta^{\prime}}$ with $\Delta^{\prime}<\Delta$. Choose a $\Delta^{\prime}$ such that $m>q$ is the number of zeros of $F(z, 0)$ in $B_{1+\Delta^{\prime}}$. Of these, $q$ zeros lie in $\partial B_{1}$ and the remaining $m-q$ in the anulus $B_{1+\Delta} \backslash \overline{B_{1}}$. Let $1+3 \delta$ be the minimum of their moduli. As the zeros depend continuously of $\tau$ there exists $\tau_{3} \in\left(0, \tau_{1}\right)$ such that, for all $\tau \in\left[0, \tau_{3}\right)$, all of their moduli are larger than $1+2 \delta$, and the same is valid for the zeros that lie in the exterior of $B_{1+\Delta}$, when $\tau=0$.

\section{Dynamic scaling behaviour}

We can now prove our main result, that generalizes Theorem III of [12].

Theorem 1. Let $c_{0} \in X^{+}$satisfy (5) and denote by $c$ the solution to (10) with initial condition $c(0)=c_{0}$. Then, the following holds true for the asymptotic behaviour of the components of $c$, 


$$
\lim _{\substack{j, t \rightarrow \infty \\ \xi=j / t \text { fixed } \\ j \in g}} t^{2} c_{j}(t)=\frac{q}{\rho} e^{-\xi / \rho},
$$

where $\mathscr{J}$ is the infinite subset of $\mathbb{N}$ given in Proposition 3 and $q=\operatorname{gcd}(P)$, with $P$ given by (12). The convergence is uniform for $\xi$ in compact intervals of $\mathbb{R}^{+}$.

Proof. Solving the initial value problems for $N(\cdot)$ and $\Phi(z, \cdot)$ given in Proposition 4 we obtain

$$
\Phi(z, t)=t^{-2} \frac{1}{N_{0}+t^{-1}} \frac{\phi(z)}{N_{0}+t^{-1}-\phi(z)}
$$

for $t>0$ and $z \in B_{1}$. Since $\Phi(\cdot, t)$ is analytic in $B_{1}$ we can apply Cauchy's integral formula and write

$$
t^{2} c_{j}(t)=\frac{1}{2 \pi i} \frac{1}{N_{0}+\tau} \oint_{\gamma_{0}} \frac{1}{z^{j+1}} \frac{\phi(z)}{N_{0}+\tau-\phi(z)} d z
$$

where $\tau=t^{-1}$ and $\gamma_{0}=\left\{z:|z|=r_{0}\right\}$ for some $r_{0} \in(0,1)$. By (5), $\phi(z)$ is analytic in $B_{1+\Delta}$ and so $\Phi(\cdot, t)$ can be extended to $B_{1+\Delta}$ as an analytic function, except for poles at the zeros of $F(z, \tau)$. By Proposition 7 there exists $\tau_{4} \in\left(0, \min \left\{\tau_{2}, \tau_{3}\right\}\right)$ such that, for all $\tau \in\left[0, \tau_{4}\right],\left|z_{k}(\tau)-\omega_{q}^{k}\right|<\delta$, for $k=1, \ldots, q$, and $\left|z_{k}(\tau)\right|>1+2 \delta$, for $k=q+1, q+2, \ldots$ Thus, if $1 \leqq k \leqq q$ we have $\left|z_{k}(\tau)\right|<1+\delta$ for all $\tau \in\left[0, \tau_{4}\right]$ and we can write

$$
\begin{aligned}
& \oint_{\gamma_{0}} \frac{1}{z^{j+1}} \frac{\phi(z)}{N_{0}+\tau-\phi(z)} d z \\
& =\oint_{\gamma_{q}+1} \frac{1}{z^{j+1}} \frac{\phi(z)}{N_{0}+\tau-\phi(z)} d z-\sum_{k=1}^{q} \oint_{\gamma_{k}} \frac{1}{z^{j+1}} \frac{\phi(z)}{N_{0}+\tau-\phi(z)} d z
\end{aligned}
$$

where $\gamma_{k}=\left\{z:\left|z-\omega_{q}^{k}\right|=\delta\right\}$ for $k=1, \ldots, q$, and $\gamma_{q+1}=\{z:|z|=1+2 \delta\}$. For sufficiently small $\delta$ the curves $\gamma_{k}$, for $k=1, \ldots, q$, are pairwise disjoint and $z_{k}(\tau)$ is the only zero of $F(\cdot, \tau)$ in the interior of $\gamma_{k}$. (If $\delta$ is such that $\gamma_{k}, k=1, \ldots, q$, are not pairwise disjoint we can choose a conveniently smaller $\delta$ and redefine $\tau_{4}$ accordingly.)

The integral over $\gamma_{q+1}$ can be estimated as in [12]: the function $F(z, \tau)$ is continuous and different from zero for $(z, \tau) \in \gamma_{q+1} \times\left[0, \tau_{4}\right]$. Thus

$$
F_{\min }:=\min _{\substack{\tau \in\left[0, \tau_{4}\right] \\|z|=1+2 \delta}}|F(z, \tau)|>0,
$$

and using (5) we have, for $\tau \in\left[0, \tau_{4}\right]$, 


$$
\begin{aligned}
\oint_{\gamma_{q}+1} \frac{1}{z^{j+1}} \frac{\phi(z)}{N_{0}+\tau-\phi(z)} d z \mid & \leqq \frac{2 \pi A}{(1+2 \delta)^{j}} \frac{1}{F_{\min }} \sum_{p_{j} \in P}\left(\frac{1+2 \delta}{1+\Delta}\right)^{p_{j}} \leqq \\
& \leqq \frac{2 \pi A}{(1+2 \delta)^{j}} \frac{1}{F_{\min }} \sum_{j=1}^{\infty}\left(\frac{1+2 \delta}{1+\Delta}\right)^{j}= \\
& \leqq \frac{2 \pi A}{(1+2 \delta)^{j}} \frac{1}{F_{\min }} \frac{1+2 \delta}{\Delta-2 \delta}
\end{aligned}
$$

Let $\xi=j \tau$. If $\xi$ is in a compact set $\left[\xi_{1}, \xi_{2}\right] \subset \mathbb{R}^{+}$we have that (24) is valid for all $j \geqq \xi_{1} / \tau_{4}$ and so the integral over $\gamma_{q+1}$ approaches 0 as $j, t \rightarrow \infty$, uniformly for $\xi$ in $\left[\xi_{1}, \xi_{2}\right]$.

The asymptotic behaviour of the other integrals can be computed using the residue theorem, l'Hôpital's rule, and (22):

$$
\begin{aligned}
-\frac{1}{2 \pi i} \sum_{k=1}^{q} \oint_{\gamma_{k}} \frac{1}{z^{j+1}} \frac{\phi(z)}{N_{0}+\tau-\phi(z)} d z & =-\sum_{k=1}^{q} \operatorname{Res}_{z=z_{k}(\tau)}\left[\frac{1}{z^{j+1}} \frac{\phi(z)}{N_{0}+\tau-\phi(z)}\right] \\
& =-\sum_{k=1}^{q} \lim _{z \rightarrow z_{k}(\tau)}\left[\frac{\phi(z)}{z^{j+1}} \frac{z-z_{k}(\tau)}{N_{0}+\tau-\phi(z)}\right] \\
& =\sum_{k=1}^{q} \frac{1}{\left(z_{k}(\tau)\right)^{j+1}} \frac{\phi\left(z_{k}(\tau)\right)}{\phi^{\prime}\left(z_{k}(\tau)\right)}
\end{aligned}
$$

To compute the limit of (25) as $\tau \rightarrow 0$ observe that

$$
\begin{aligned}
\lim _{\tau \rightarrow 0} \phi\left(z_{k}(\tau)\right) & =\lim _{\tau \rightarrow 0}\left(N_{0}+\tau-F\left(z_{k}(\tau), \tau\right)\right)=\lim _{\tau \rightarrow 0}\left(N_{0}+\tau\right)=N_{0} \\
\lim _{\tau \rightarrow 0} \phi^{\prime}\left(z_{k}(\tau)\right) & =\lim _{\tau \rightarrow 0}\left(z_{k}(\tau)\right)^{-1} \sum_{p_{j} \in P} p_{j} c_{0 p_{j}}\left(z_{k}(\tau)\right)^{p_{j}} \\
& =\omega_{q}^{-k} \sum_{p_{j} \in P} p_{j} c_{0 p_{j}} \omega_{q}^{p_{j} k} \\
& =\omega_{q}^{-k} \rho
\end{aligned}
$$

and

$$
\lim _{\tau \rightarrow 0}\left(z_{k}(\tau)\right)^{j+1}=\omega_{q}^{k} \lim _{t \rightarrow 0}\left[\omega_{q}^{k}\left(1+\frac{1}{\rho} \tau+R^{(2)}(\tau)\right)\right]^{j}
$$

if $j \in \mathscr{J}$ then 


$$
j=\sum_{i} n_{i, j} p_{i}=\sum_{i} n_{i, j} m_{i} q=v_{j} q
$$

with $v_{j}=\sum_{i} n_{i, j} m_{i}$. This implies that $\left(\omega_{q}^{k}\right)^{j}=\left(\omega_{q}^{q}\right)^{v_{j} k}=1$. If, additionally, $\xi=j \tau$ is fixed, the limit in the right-hand side of (28) can be written as

$$
\lim _{j \rightarrow \infty}\left(1+\frac{\xi / \rho}{j}+R^{(2)}(1 / j)\right)^{j}
$$

which is equal to $e^{\xi / \rho}$.

Using this result, together with (26) and (27), we can compute the limit of (25) as $j \rightarrow \infty$ and $\tau \rightarrow 0$, with $\xi=j \tau$ fixed and $j \in \mathscr{J}$, obtaining (23).

Acknowledgement. I am grateful to Luísa Ribeiro for helpful discussions.

\section{REFERENCES}

1. J. M. BALl and J. CARR, The discrete coagulation-fragmentation equations: existence, uniqueness, and density conservation, J. Statist. Phys. 61 (1990), 203-234.

2. J. M. Ball, J. CARr and O. Penrose, The Becker-Döring cluster equations: basic properties and asymptotic behaviour of solutions, Comm. Math. Phys. 104 (1986), 657-692.

3. J. M. Ball, P. J. Holmes, R. D. James, R. L. Pego and P. J. Swart, On the dynamics of fine structure, J. Nonlinear Sci. 1 (1991), 17-70.

4. J. CARr and F. P. DA Costa, Instantaneous gelation in coagulation dynamics, Z. Angew. Math. Phys. 43 (1992), 974-983.

5. J. Carr and F. P. DA Costa, Asymptotic behavior of solutions to the coagulationfragmentation equations. II. Weak fragmentation, J. Statist. Phys. 77 (1994), 89-123.

6. F. P. DA Costa, On the positivity of solutions to the Smoluchowski equations, Mathematika 42 (1995), 406-412.

7. R. Drake, A general mathematical survey of the coagulation equation, in G. M. Hidy and J. R. Brock (eds.), Topics in Current Aerosol Research (part 2) (International Reviews in Aerosol Physics and Chemistry, Pergamon Press, Oxford, 1972).

8. M. H. Ernst and P. G. van Dongen, Scaling solutions of Smoluchowski's coagulation equations, J. Statist. Phys. 50 (1988), 295-329.

9. F. Family and T. VicseK, Dynamic scaling for aggregation of clusters, Phys. Rev. Lett. 52 (1984), 1669-1672.

10. S. Friedlander and C. WANG, The self-preserving particle size distribution for coagulation by brownian motion, J. Coll. Interface Sci. 22 (1966), 126-132.

11. L. K. HuA, Introduction to Number Theory (Springer-Verlag, Berlin, 1982).

12. M. Kreer and O. Penrose, Proof of dynamic scaling in Smoluchowski's coagulation equation with constant kernels, J. Statist. Phys. 74 (1994), 389-407.

13. M. Shirvani and H. van RoEssel, The mass-conserving solutions of Smoluchowski's coagulation equation: the general bilinear kernel, Z. Angew. Math. Phys. 43 (1992), 526-535. 
14. M. Slemrod, Trend to equilibrium in the Becker-Döring cluster equations, Nonlinearity 2 (1989), 429-443.

15. W. White, A global existence theorem for Smoluchowski's coagulation equation, Proc. Amer. Math. Soc. 80 (1980), 273-276.

\author{
InSTITUTO SUPERIOR TÉCNico \\ Departamento de Matemática \\ Av. Rovisco Pais \\ P-1096 LISBOA \\ Portugal \\ E-mail address: fcosta@math.ist.utl.pt
}

